\title{
LA MÉCANISATION DES EXPLOITATIONS FORESTIERES
}

\section{Difficultés - Solutions}

\section{I. - Les difficultés}

Depuis quelques années, les exploitations forestières se sont mécanisées. D'incontestables progrès ont été faits dans l'outillage et les méthodes de travail. Les ouvriers se sont habitués aux machines et ont appris à s'en servir. Beaucoup d'ouvriers, qui travaillent encore à la main, souhaitent maintenant vivement acheter des machines mais hésitent, pour des raisons diverses, à la lumière des difficultés que la mécanisation rencontre pour être parfaitement au point et rentable.

Quelles sont ces difficultés et quelles solutions peuvent être envisagées pour les pallier?

Les difficultés de la mécanisation des exploitations forestières résultent de quatre groupes de causes:

- la station,

- la nature di peuplement,

- l'état actuel de la main-d'œuvre,

- la diversité des produits façonnés.

\section{I) Difficultís Résultant de la station}

Ces difficultés tiennent à l'éloignement des exploitations par rapport aux villages, à la pente, au sol et aux intempéries.

Malgré l'extension régulière du réseau routier forestier, l'accès aux coupes représente toujours un long trajet à faire à pied: en moyenne 3 à 5 kilomètres, en montagne. En outre, la coupe est loin du domicile du bûcheron, d'autant plus que les écarts sont peu à peu abandonnés au profit du village où règnent la plupart des ( bienfaits ) de la civilisation. Or, l'outil mécanique est lourd et il a besoin de carburant, huile et outillage. Tout ceci ne peut guère être transporté à pied ni même à bicyclette.

Une grande partie des forêts françaises se trouvent en montagne, la pente constitue un second obstacle à la mécanisation. Il n'est pas rare, dans les Alpes, de voir des forêts sur des pentes de plus de I00 \% (J'ai relevé I $33 \%$ près de Barcelonnette, dans un mélézin). 
Le parcours y est difficile pour un forestier, à plus forte raison pour un bûcheron portant une scie de $40 \mathrm{~kg}$. En outre, si l'on veut abattre un arbre vers le haut de la pente (ce qui est réglementaire), même si l'entaille est faite bien au ras du sol, côté amont, le trait' de scie de chute, côté aval, doit parfois être exécuté avec l'outil tenu à bout de bras. La légèreté de cet outil est donc une conidition importante.

Le sol, par sa nature et son humidité, constitue un troisième élément de difficulté. D'abord parce qu'il rend souvent la circulation difficile, du fait qu'il est collant, glissant, détrempé ou encombré de rochers. Mais aussi parce que les cailloıx qu'il contient, accrochés par les dents des scies ont vite fait de les désaffûter. On s'en aperçoit rapidement avec les circulaires coupant au ras du sol.

Les intempéries rendent déjà difficile le travail à main (il ne faut pas oublier que la plupart des exploitations ont lieu en hiver); elles constituent pour le travail mécanique une gêne sérieuse. Le démarrage, à froid, des moteurs à explosion n'est pas toujours facile. A11 moment des pleins ou des nettoyages, des gouttes d'eau peuvent arriver dans les réservoirs et carburateurs. Un outil mécanique traînant sous la pluie, dans la neige ou dans la boue arrive vite à s'endommager, car la protection des organes fragiles n'est pas toujours excellente.

Eloignement, pente, sol et intempéries posent aussi les mêmes difficultés pour la vidange des produits. Toutefois, le tracteur ou le camion tous terrains présentent sur l'attelage certaines supériorités: plus grande vitesse, puissance plus élevée, organes d'adhérence, treuils, qui dans de nombreux cas facilitent et accélèrent le travail. A noter aussi la multiplication des câbles aériens pour le transport des grumes ou des petits bois qui résolvent les problèmes de transport les plus difficiles.

\section{2) Difficultés résultant de la NATURE DU PEUPlement}

Ces difficultés proviennent:

d'une part, des caractéristiques des arbres à abattre,

d'autre part, de l'obligation de respecter le peuplement réservé, les semis ou l'ensauchement.

\section{a) Difficultés provenant des caractéristiques des arbres à abattre.}

Les arbres à abattre ont souvent des dimensions très variées, une forme plus ou moins irrégulière, des branches plus ou moins nombreuses, un bois de dureté variable et ils sont plus ou moins dispersés au sein du peuplement réservé.

La variété dans les dimensions est extrême dans les coupes de 
jardinage. Mais il y a aussi des coupes de régénération où l'on doit abattre des gros arbres et recéper des petits bois dans les fourrés. Dans les taillis-sous-futaie, il y a deux types de peuplement à exploiter et de nombreuses classes de diamètre. La population d'un taillis, même très régulier d'apparence. comme un taillis de châtaignier, se compose de brins dont le diamètre varie de $\mathrm{d} / 3$ à $3 \mathrm{~d}$ (si $\mathrm{d}$ est le diamètre moyen). Enfin, dans une éclaircie de futaie régulière, on enlève à la fois des brins dominés et des tiges dominantes de grosseurs très différentes. Il n'est donc pas toujours possible de faire le travail avec un seul outil mécanique. On a le choix alors entre plusieurs solutions: Avoir deux scies d'abattage, l'une pour les petits bois, l'autre pour les gros bois (et peut-être en plus une tronçonneuse à petits bois) : cela coûte cher. Prendre une scie à gros bois avec laquelle on coupera aussi les petits, mais, à cause du poids élevé, on perdra du temps. Utiliser un outil moyen, mais celui-ci peinera avec les tiges les plus fortes.

La forme des tiges gêne parfois le travail à la machine: pieds tordus des perches de taillis de charme ou de chêne, empattement très développé de certains arbres, flexuosité des fûts qui les fait pencher du côté opposé à celui où l'on doit les abattre et ralentit le trait sciage d'abattage (les chronométrages donnent à cet égard des résultats très dispersés).

La dureté $d u$ bois est très variable, parce que les essences sont mélangées ou parce que le bois des branches (rais cle sapin) est bien plus dur que celui des tiges ou parce que le bois est gelé. Le bûcheron vosgien possède deux haches: la hache "charbonnière ) et la hache de " rais ». Avec les machines, il faut aussi apporter sur la coupe des chaines de rechange de plusieurs types de denture. L'ébranchage à la scie mécanique n'est d'ailleurs pas toujours facile à cause de la forme des branches et de leur disposition, en plus de leur plus grande dureté.

La dispersion des arbres à abattre, au sein du peuplement réservé constitue aussi un sérieux obstacle: le temps de déplacement devenant aussi long (ou plus long) que le temps de travail. Si la coupe à blanc étoc de plantations est particulièrement facile à mécaniser, l'organisation d'un chantier mécanisé dans une éclaircie ou dans une coupe de régénération n'est pas toujours facile. Ceci constitue en particulier un sérieux obstacle à l'utilisation de groupes électrogènes avec scies munies de moteurs électriques, solution qui est excellente pour les coupes à blanc-étoc. Le groupe électrogène présenterait cependant de grands avantages: un seul moteur à explosion, plusieurs prises de courant sur lesquelles on peut brancher des outils variés, outils plus légers, plus maniables, moins de vibrations, pas de gaz d'échappement nocifs. Cette solution qui est très répandue dans certains autres pays, n’a pas été assez étudiée chez nous, 


\section{b) Difficultés provenant du peuplement réservé.}

Le respect du peuplement réservé constitue aussi une sérieuse sujétion pour le bûcheron, qu'il s'agisse d'arbres réservés ou de taches rle semis. Malgré la virtuosité incontestable de certains bûcherons, j'ai toujours constaté qu'il était plus difficile de guider la chute à la machine qu'à la main. L'utilisation de palans genre Tirefor pallie, dans une certaine mesure, cette infériorité. Mais il est fréquent de voir des bûcherons mécanisés abattre avec des outils à main les arbres les plus difficiles de leur lot.

A noter en outre, en ce qui concerne le peuplement réservé, que l'ensouchement du taillis constitue une sorte de réserve. La coupe rez terre et en dôme, dans une cépée dense où l'on ne peut pas faire pénétrer la scie mécanique, pose de sérieuses difficultés, car l'abattage serait plus facile et la souche mieux faite si la perche pouvait être sciée par l'intérieur de la cépée.

Dans ce qui précède, il n'a été parlé que de l'abattage, mais la variété des dimensions des arbres à abattre, leurs irrégularités de forme, leur dispersion, la présence d'arbres réservés, de semis ou de souches à respecter, rendent difficiles également la vidange et imfosent des sujétions pour sa mécanisation (largeur maxima des tracteurs, exclusion des chenilles, obligation d'employer des engins de traction ou de roulage, etc...).

Enfin, on peut ranger parmi les impératifs sylvicoles la question cics délais pour l'abattage, le façonnage ou la vidange. Mais ici la mécanisation, en hâtant le travail, peut faciliter l'observation de ces délais et même les raccourcir.

\section{3) Difficultés résultant de l'État actuel DE LA MAIN-D'EUVRE}

La main-d'wuvre forestière vicillit et se raréfie. Le problème est rural, il n'est pas spécial à la forêt. En outre, le bîcheron n'est généralement pas un professionnel, mais un petit agriculteur ou un uuvrier agricole qui consacre à la forêt une partie de son temps, le partage n'étant pas seulement saisonnier, mais aussi journalier.

Ceci ne lui permet guère d'améliorer sa formation professionnelle.

Cependant, on voit des bûcherons, même saisonniers, qui se décident à acheter des outils mécaniques. Certains reconnaissent même que, grâce à leur scie mécanique, ils peuvent gagner plusieurs heures par jour pour " travailler à la maison ».

Enfin, cette main-d'œuvre est souvent démunie de noyens financiers. Or, une bonne scie mécanique, avec chaînes de rechange et appareil affûteur, représente une mise de fonds de près de 200000 francs.

Le bûcheron travaillera donc comme tâcheron, à tant du mètre 
cube ou du stère, pour un patron qui n'est guère poussé à acheter des scies mécaniques à ses ouvriers et, en général, ne s'occupe pas de l'amélioration de leur formation professionnelle.

\section{4) Difficultés résultant de la diversité DES PRỌDUTS FAÇONNÉS}

La variété des diamètres cause des difficultés pour une bonne organisation d'un chantier mécanisé d'abattage. Il en est de même en ce qui concerne le façonnage à cause de la diversité des produits à préparer.

Le façonnage, en effet, s'accompagne d'un classenient avant, pendant ou après le tronçonnage; ce classement oblige à considérer l'essence, les dimensions, la forme et les qualités des perches; il ralentit donc considérablement l'opération de façonnage.

En outre, l'industrie évolue; elle utilise des produits nouveaux ou présentés de façon nouvelle avec des cahiers des charges qui se précisent (ce qui est nécessaire) mais se compliquent (ce qui est fatal).

\section{a) Sujétions lićes au classement des produits}

Il est très facile de déliter des bûches de bois de feu ou des rondins de papeterie ayant uniformément un mètre de longueur. Il n'en est pas cle même quand il s'agit de sélectionner, parmi les perches, des bois de mine répondant à des caractéristiques précises de dimensions, de forme et de qualité. Dans bien des coupes de taillis ou d'éclaircie, le médiocre rendement des machines à tronçonner provient dı temps passé pour classer (et même marquer) les bois avant façonnage. Si on marque les perches sur le sol, avant tronçonnage (dans le cas où, par exemple, le tronçonnage n'est pas fait sur place, mais après manutention ou clébardage), il arrive fréquemment que les marques soient placées clu mauvais côté en arrivant sous la tronçonneuse, ce qui amène une perte de temps. Si c'est le tronçonneur qui fait le classement tout en sciant, son outil ne travaille que pendant une fraction infime du temps total.

En outre, la mécanisation accélère certaines phases de l'exploitation (tandis que certaines phases restent obligatoirement manuelles) et l'organisation des chantiers s'en trouve quelque peu bouleversée. J'ai vu dans certaines coupes, d'habiles et actifs bûcherons mécanisés venir abattre quelques arbres puis s'en aller dans une autre coupe pendant que d'autres ouvriers faisaient l'ébranchage manuel. Ensuite, quand le marchand de bois avait tracé ses découpes, c'est-à-dire effectué l'opération de classement, les ouvriers revenaient faire le tronçonnage à la machine. Puis quand le débarcleur avait enlevé les grumes, ils revenaient à nouveau pour tronçonner les brançhes. Ceci amène façilement des pertes de temps et 
d'argent qui conduisent certains bûcherons à reculer devant la mécanisation.

b) Sujétions liées à l'apparition de nouveaux débouchés.

Enfin, les cahiers des charges relatifs aux nouveaux produits ou aux nouvelles présentations de certains produits et les progrès de la technique provoquent l'apparition de travaux particuliers de façonnage qui compliquent l'organisation des chantiers mécanisés; par exemple, la mise au point d'écorceuces mécaniques pour feuillus de petites dimensions et de forme nettement plus irrégulière que celle des résineux. Or, cette opération d'écorçage, particulièrement difficile, doit, elle aussi, se mécaniser pour se synchroniser avec l'abattage et le tronçonnage mécaniques.

L'emploi de produits de préservation pour les bois à fibres feuillus et les grumes à essences altérables pose les mêmes problèmes de transport et de stockage des outils et des produits que les scies à moteur. En ce qui concerne les bois altérables, la mécanisation qui permet d'aller vite en choisissant des époques favorables, se révèle d'ailleurs particulièrement utile.

Dans l'ensemble, les conditions de travail en forêt ont donc rendu jusqu'à ce jour, assez difficile l'extension de la mécanisation. Or, cette mécanisation est fatale. Elle répond à la nécessité de diminuer les efforts physiques des hommes et d'améliorer leur condition. En outre, la mécanisation est contagieuse. De l'usine, elle a gagné la ferme, puis la forêt. Le tracteur a entraîné dans son sillage la scie mécanique d'abattage et de façonnage. Puis, se tournant vers les phases du travail non encore entièrement mécanisées, par exemple l'ébranchage ou l'écọrçage, l'homme cherche à étendre toujours la mécanisation pour diminuer sa peine et augmenter son salaire. Tout ceci est parfaitement juste. Il ne faut pas se borner à nier les avantages de la mécanisation ou à contester sa rentabilité. Il serait inadmissible de s'opposer à ses progrès en forêt. Il importe donc de rechercher comment la machine, bien mise au point, confiée à des ouvriers ayant reçu une formation professionnelle nécessaire, intégrée dans une équipe où l'organisation du travail et la sécurité ont été étudiées, peut accomplir les diverses tâches du travail forestier, tout en répondant aux impératifs sylvicoles que nous devons défendre et aux sujétions d'ordre économique (prix de revient admissible et qualité maxima des produits façonnés).

\section{II. - Les solutions}

Pour faciliter l'extension de la mécanisation des travaux forestiers, deux groupes de solutions doivent être envisagées:

- des solutions forestières.

- des solutions techniques. 


\section{I) Solutions FORESTIÈres}

Il s'agit de s'efforcer de pallier une partie des difficultés exposées ci-dessus, résultant des caractéristiques des arbres à abattre et de la présence de réserves à respecter.

Un certain nombre de techniciues, qui ont été employéés chez nous sur une grande échelle, répondent d'ailleurs à cette sujétion. L'enrésinement des taillis favorisera à échéance plus ou moins lointaine la mécanisation. La conversion des taillis et des taillis-sousfutaie en futaie régulière, offrant aux bûcherons des produits bien calibrés et des tiges nettement séparées les unes des autres. non groupées en cépées, facilite également le travail à la machine. Le raccourcissement des révolutions dans les forêts où l'on ne recherche' pas certaines qualités exceptionnelles liées à l'âge et à la grosseur, faciliterait également l'abattage et le tronçonnage mécaniques. Enfin, dans les coupes où il y a deux types de peuplements à exploiter. il serait intéressant que l'exploitation puisse être faite en deux temps. Par exemple, dans une coupe secondaire, ne pas vendre ou exploiter en même temps la coupe de gros bois et le nettoiement des gaulis.

L'amélioration des produits sur pied peut aussi, dans une certaine mesurer, faciliter la tâche du bûcheron. Par exemple, l'élagage artificiel des peuplements résineux rend bien plus facile l'ébranchage au moment de l'abattage et permet d'intégrer cette opération, jusqu'ici le plus souvent manuelle, dans le travail d'une équipe dotée d'engins mécaniques d'abattage et de tronçonnage.

Les parcellaires actuels ne sont pas toujours compatibles avec une exploitation mécanisée, surtout en ce qui concerne le débardage. Les limites de parcelles sont souvent des lignes droites tracées arbitrairement, par monts et par vaux, et qui ne peuvent servir de voies de vidange. L'utilisation de chemins de vidange comme limites de parcelles est toujours préférable. Beaucoup de chemins de bas de pente constituent en outre des limites écologiques et séparent des peuplements distincts qu'il n'est plus rentable de vendre et d'exploiter séparément. Nous en avons vu d'intéressants exemples dans certaines forêts de l'est. En montagne, où l'on est amené à utiliser des câbles faute de routes, il y a lieu également de songer que le parcellaire doit permettre l'installation d'un tracé de câble jusqu'à une route camionnable.

Le réseau routier s'est considérablement amélioré. Mais souvent encore il y a lieu de l'adapter à la largeur et au rayon de braquage des véhicules de transport actuels en particulier des semi-remorques de 20 tonnes, de plus en plus employées. Enfin, la route ne doit pas s'arrêter au seuil de la parcelle, mais se prolonger et se ramifier à l'intérieur par un réseau de chemins et de pistes, semi-permanents, pour véhicules porteurs à deux essieux-moteurs ou pour 
tracteurs avec remorques ou triqueballes. Ces chemins doivent être explicitement repérés et entretenus pendant la période des exploitations. Tous les types de véhicules doivent y être acceptés, mais avec une interdiction expresse pour ceux-ci d'en sortir et de circuler dans la coupe. Quand cette précaution n'est pas' prise, les tfacteurs vagabondent au travers des coupes au hasard de fourrés qui leur semblent moins rébarbatifs (souvent parce qu'ils sont moins épineux) ou en profitant des trouées faites par la chute des arbres, ce qui cause de graves dégâts et l'application des. sanctions prévues par la loi. Ce réseau semi-permanent de pistes et chemins, à l'intérieur des parcelles, sert en même temps à “ placer » les cimes des arbres à abattre sans endommager les semis. Mais il n'existe pas partout. Ancrés sur ces chemins, les tracteurs-treuils peuvent hâler à eux le grumes par trainage sur le sol nu ou au moyen de pelles ou de cônes de débardage, sans grands dommages pour le peuplement, surtout si en abattant ses arbres, le bûcheron a eu le soin de les orienter dans le bon sens (gros bout vers l'avant).

Un certain nombre de mesures légales seraient souhaitables pour faciliter lẹ développement de la mécanisation. L'interdiction de l'usage de la scie pour l'abattage des taillis s'applique-t-elle à la scie mécanique? L'abattage et l'écorçage en sève de certains feuillus viennent d'être autorisés. Les conditions relatives à l'emploi des tracteurs pourraient être assouplies. L'avancement de la date des ventes serait éminemment utile, car cela permettrait de commencer les exploitations dans la deuxième quinzaine de septembre, bien avant la neige. On peut remarquer que les hêtres abattus avant et au début de l'hiver, se conservent beaucoup mieux que ceux qui sont abattus en mars, avril, mai et même fin février. Il ne serait pas impossible, de cette manière, surtout grâce à la mécanisation et à la livraison rapide, devenue habituelle; de certains produits, que les coupes commencées en octobre soient totalement débardées et vidangées l'été suivant.

Mais tout ceci constitue un ensemble de mesures forestières que nous n'avons pas encore étudié de façon détaillée et nous préférons donner davantage de précisions sur les solutions techniques.

\section{2) Solutions techniques}

Les diverses solutions techniques destinées à faciliter l'extension de la mécanisation des exploitations forestières peuvent se grouper comme suit:

- anélioration de la main-d'œuvre,

- amélioration de l'outillage,

- amélioration des conditions de travail. 


\section{a) Amélioration de la main-d'œuvre}

La main-d'œuvre forestière présente certaines faiblesses que nous avons exposées plus haut. Il n'est pas toujours possible d'en faire une main-d'œuvre absolument' permanente, car il est logique que le bûcheron, demeurant à la campagne, veuille subvenir à ses besoins par un peu de culture et d'élevage. Mais si la forêt peut lui assurer un travail rémunérateur pendant la majewre partie de l'année, la culture ne sera plus pour lui qu'un appoint (et non l'inverse comme c'est le cas actuellement). Le bûcheron doit en même temps être ouvrier sylviculteur. Ceci est facile à réaliser dans les forêts où les travaux de sylviculture et l'exploitation des coupes sont faits en régie sous le contrôle du propriétaire de la forêt. Ce système réussit parfaitement en Alsace et en Lorraine, dans les forêts soumises et dans bon nombre de forêts privées, à la satisfaction d'ailleurs des industriels du bois qui peuvent alors se consacrer à leur tâche, de plus en plus difficile, d'industriels. C'est dans les régions où l'exploitation des coupes se fait en régie que la mécanisation s'est répandue le plus rapidement et le plus intensément.

Le métier de bûcheron-sylviculteur, qui constitue un véritable métier, est évidemment plus difficile. En effet, non seulement le travail du bûcheron mécanisé est devenu plus compliqué que celui du bûcheron manuel, mais le travail du sylviculteur (pépinières, plantation, dégagement des semis, entretien des routes) nécessite une grande compétence et une longue formation à cause du caractère saisonnier des travaux.

La formation professionnelle dans ce domaine est donc indispensable. De même que la formation professionnelle a créé dans les usines une classe d'ouvriers spécialisés, à niveau de vie amélioré, la formation professionnelle améliore à la fois la condition des ouvriers forestiers, leur rendement et la qualité de leur travail. La forêt et les produits forestiers y gagneront.

La question de la formation professionnelle fait l'objet dans ce numéro de la Revue forestière d'un article de M. l'Ingénieur KoвLOTH. Je n'insisterai donc pas.

En même temps qu'une formation professionnelle complète, il faut aussi donner aux ouvriers forestiers une formation sociale et en particulier leur enseigner les règles indispensables sur l'hygiène et la sécurité du travail. Ceci fait d'ailleurs l'objet à l'Ecole de bûcherons de Saverne de cours et exercices pratiques très documentés.

A côté de la formation de bûcherons et de sylviculteurs, il y aurait lieu également de songer à la formation professionnelle des conducteurs de tracteurs et des câblistes, formation qui intéresse tout autant le propriétaire de forêt que l'exploitant.

'Il est à noter que la formation professionnelle, telle qu'elle est 
conçue ne vise pas seulement à améliorer les bûcherons actuels, mais à former de jeunes éléments qui seront alors pourvus d'un Certificat d'Aptitude Professionnelle, clef d'un véritable métier. Une bonne formation professionnelle, un outillage mécanisé et les conséquences qui en résultent sur l'amélioration de la condition ramèneront vers la forêt les jeunes qui y sont devenus rares. Ce mouvement s'est déjà nettement esquissé dans l'est de la France.

I_e Certificat d'Aptitude Professionnelle devrait pouvoir servir également, comme cela se fait dans certains pays, à permettre aux jeunes ouvriers d'obtenir l'aide financière nécessaire pour acquérir lour premier outillage mécanisé et le moyen de transport motorisé individuel qui le complète.

\section{b) Amélioration de l'outillage}

Dans un autre article de ce numéro de la Revue forestière, M. H. Duterl, Assistant à la 4 Section de la Station de Recherches, expose quelques-uns des résultats obtenus sur les chantiers expérimentaux de la Section.

L'outillage mécanisé d'abattage, tel qu'il se présentait il y a quelquesl années, comportait de graves défauts : lourd, encombrant, sujet à de nombreuses pannes, manquant d'efficacité et coûteux. Il y avait de quoi rebuter des ouvriers même d'esprit novateur et ayant les ressources financières suffisantes.

Mis au ban d'essai de la forêt, après avoir été conçus dans un atelier et éprouvés dans une cour d'usine, les outils mécaniques ont révélé leurs défauts et, grâce à la cordiale collaboration qui s'est créée entre bûcherons, mécaniciens des usines et personnels des organismes de recherches, les améliorations à réaliser se sont précisées. Les outils mécaniques ont été mis au point peu à peu, en France et dans les pays voisins. Les organismes internationaux qui diffusent de façon diligente les documents et les notes techniques des divers pays ont d'ailleurs grandement facilité cette amélioration de l'outillage. Des outils mécaniques étrangers ont pu être expérimentés dans nos forêts, côte à côte avec des outils français et des butils manuels, dans les mêmes conditions de travail et avec la même main-d'œuvre. Les défauts et les qualités de chacun ont donc pu être mis en relief.

On trouvera à ce sujet, dans l'article de M. DuteIL des renseignements détaillés. Evidemment, bien des progrès restent à faire en matière d'outillage mécanisé et les constructeurs de machines ne veulent pas toujours l'admettre. Il s'agit parfois de petits perfectionnements qui peuveunt paraitre futiles (système d'armature à dos pour le transport, courroies de suspension pour le travail) ou dont l'efficacité est controversée (chaine à crochets, plus facile à affûter, tréteaux à rouleaux amenant les perches sous les machines 
à tronçonner, blindages de protection des pièces délicates, tubulures d'échappement éloignant les gaz, etc...) ou encore des petites mises au point (amélioration du graissage, diminution de la faiblesse mécanique de certaines pièces).

La question est parfois plus grave, par exemple quand il s'agit de déterminer le type de scie convenant pour un peuplement d'essence êt de diamètre donnés. Les petites scies sont plus économiques et plus légères, mais elles renâclent devant un diamètre trop fort, surtout si l'arbre " pèse " sur la lame coupante à cause de la forme de sa cime. Les grandes scies coûtent plus cher, sont lourdes et consomment plus, mais elles viennent plus facilement à bout des difficultés. On constate par exemple que la surface sciée à l'unité de temps en fonction du diamètre des perches atteint rapidement un maximum avec les petits outils et décroit très vite. La scie mécanique ne doit jamais travailler à la limite supérieure de sa puissance.

Nous sommes sceptiques également en ce qui concerne les outils à tout faire. Les diverses phases du travail forestier sont très différentes et les meilleurs rendements sont obtenus avec les outils spécialisés. Leur inconvénient actuel est la nécessité de doter chacun d'eux d'un moteur à explosion individuel. C'est pourqubi la solution du groupe électrogène avec 2 ou 6 prises de courant nous semble préférable.

Mais le problème le plus difficile est l'amélioration de l'Organisation du travail sur les chantiers mécanisés. L'outil n'est rien sans l'homme. Des fautes d'organisation amènent une chute verticale du rendement. Nous en avons relevé un certain nombre au cours de nos chronométrages de chantiers. L'organisation est d'autant plus difficile que la présence de machines nécessite généralement la création d'équipes. Ce sont d'ailleurs des équipes qui, les premières à frais communs ont acheté des machines. Toutefois, certains ouvriers préfèrent travailler seuls. Nous en avons chronométré d'intéressants exemples. Mais, malgré leur bon rendement et leur excellent travaii, ces ouvriers ne tirent pas le meilleur parti de leurs machines.

Dans les équipes, il y a lieu de procéder à une spécialisation du travail (tout au moins à une spécialisation temporaire, avec relais, le changement périodique constituant un repos). Les machines et ceux qui les servent ne doivent pas attendre. Le problème des temps perdus en allées et venues est d'ailleurs plus difficile à résoudre sur les chantiers mécanisés à cause du poids des machines et de leur approvisionnement en carburant. Par ailleurs, l'affûtage et l'entretien ne doivent pas interrompre le travail. Ce problème d'organisation en équipes qui paraissait irréalisable dans de nombreuses régions àvec les ouvriers manuels semble se résoudre plus facilement autour d'une machine achetée à frais communs (ou achetée par un entrepreneur qui travaille avec ses ouvriers, comme chef d'équipe et 
quii s'occupe de l'entretien et de l'affûtage.). Nous avons été très favorablement impressionnés par l'esprit d'entreprise et d'équipe qui régnait au sein de certains chantiers mécanisés, assez équiennes d'ailleurs. La formation professionnelle achèvera de créer cét esprit de travail en conmuni, éminemment favorable au rendement.

\section{c) Amélioration des conditions de travail}

La mécanisation amène de nouvelles servitudes. par exemple celle du transport des outils et du carburant vers la coupe. Le bûcheron mécanisé a donc tendance à se motoriser. L'isolé a son vélomoteur avec remorque pour l'outillage. L'équipe a souvent son automobile, généralement rustique, mais presque " tous terrains ". Ce côté de l'aménagement des chemins forestiers n'est pas à négliger: création de pistes motocyclables, nivellement de certains chemins pour les rendre praticables aux Jeeps ou à leurs ersatz.

L'outil mécanique craint les intempéries prolongées. plus encore que l'homme. La baraque démontable et transportable destinée à abriter les ouvriers devient encore plus utile quand il faut stocker pour la nuit, les scies, les bidons de carburant et abriter une petite machine à affûter les chaines. Dans la baraque, on place également la malette de pansements, au service de l'équipe, accessoire qui a toujours été utile mais souvent négligé. Et l'équipe trouve dans sa baraque un abri contre les intempéries de courte durée et un lieu favorable pour son repas de midi, côté psychologique non négligeable.

Nous croyons et nous répétons que la mécanisation est fatale, ce mot étant pris sans aucune pensée de regret, bien au contraire, car elle seule permettra l'amélioration de la condition des ouvriers forestiers.

Mais cette mécanisation nécessite le concours des forestiers qui, tout en sauvegardant l'avenir de la forêt, leur tâche essentielle, doivent s'employer à faciliter le travail mécanique. Il leur revient aussi d'assurer techniquement la formation professionnelle de la maind'œuvre et de créer, pour cette main-d'œuvre les meilleures conditions de travail; ce qui a été fait à cet égard, depuis quelques années, dans les trois Conservations d'Alsace et de Lorraine, doit servir d'exemple.

\section{J. VENET,}

Ingénieur Principal des Eaux et Forêts, Chef de la $4^{\circ}$ Section de la Station de Recherches Forestières. 